\title{
BMJ Open Non-pharmacological interventions to improve the patient experience of colonoscopy under moderate or no sedation: a systematic review protocol
}

\author{
Colin Sue-Chue-Lam (D , ,, Matthew Castelo, ${ }^{1,2}$ Jill Tinmouth, ${ }^{2,3}$ Diego Llovet, ${ }^{2,4}$ \\ Teruko Kishibe, ${ }^{5}$ Nancy N Baxter ${ }^{2,6}$
}

To cite: Sue-Chue-Lam C, Castelo M, Tinmouth J, et al. Non-pharmacological interventions to improve the patient experience of colonoscopy under moderate or no sedation: a systematic review protocol. BMJ Open 2020;10:e038621. doi:10.1136/ bmjopen-2020-038621

- Prepublication history and additional material for this paper are available online. To view these files, please visit the journal online (http://dx.doi. org/10.1136/bmjopen-2020038621).

Received 18 March 2020 Revised 12 August 2020 Accepted 17 August 2020
Check for updates

(C) Author(s) (or their employer(s)) 2020. Re-use permitted under CC BY-NC. No commercial re-use. See rights and permissions. Published by BMJ.

For numbered affiliations see end of article.

Correspondence to

Dr Nancy N Baxter;

BaxterN@smh.ca

\section{ABSTRACT}

Introduction The patient experience is a critical dimension of colonoscopy quality. Sedative and analgesic drugs are commonly used to improve the patient experience of colonoscopy, with predominant regimens being deep sedation, typically achieved with propofol, and moderate sedation, typically achieved with an opioid and a benzodiazepine. However, non-pharmacological interventions exist that may be used to improve patient experience. Furthermore, by identifying nonpharmacological interventions to increase the quality of patient experience under moderate sedation, jurisdictions facing rising use of deep sedation for colonoscopy and its significant associated costs may be better able to encourage patients and clinicians to adopt moderate sedation. Advancing either of these aims requires synthesising the evidence and raising awareness around these non-pharmacological interventions to improve the patient experience of colonoscopy.

Methods and analysis A systematic review will be conducted that searches multiple electronic databases from inception until 2020 to identify randomised controlled trials evaluating what, if any, non-pharmacological interventions are effective compared with placebo or usual care for improving the patient experience of routine colonoscopy under moderate or no sedation. Two reviewers will independently perform a three-stage screening process and extract all study data using piloted forms. Study quality will be assessed using the Cochrane Risk of Bias Tool V.2.0. Where multiple studies evaluate a single intervention, evidence will be quantitatively synthesised using pairwise meta-analysis, otherwise narrative syntheses will be undertaken.

Ethics and dissemination This is a review of existing literature not requiring ethics approval. The review findings will be included in future efforts to develop an implementation strategy to reduce the use of deep sedation for routine colonoscopy. They will also be published in a peer-reviewed journal, presented at conferences and contribute to a doctoral thesis. PROSPERO registration number CRD42020173906.

\section{INTRODUCTION}

Across the USA and Canada, outpatient, lowrisk ('routine') colonoscopy is increasingly
Strengths and limitations of this study

- The patient experience is a critical dimension of colonoscopy quality, and this review seeks to aid knowledge users in improving the patient experience of colonoscopy

- The proposed review is designed and will be reported in accordance with best practice guidelines,

- Two independent reviewers will conduct all screening and data extraction, contacting authors for data clarification and including a third reviewer to resolve disagreements if needed,

- Heterogeneity in the interventions and outcomes used in existing studies may pose a barrier to quantitative synthesis.

being performed under 'deep sedation' with propofol rather than 'moderate sedation' with opioids and benzodiazepines. ${ }^{1-3}$ It is clear that patient experience is an important dimension of colonoscopy quality, ${ }^{4-6}$ that sedation improves patient experience and colonoscopy effectiveness, and that deep sedation may be particularly valuable for prolonged procedures, emergency procedures and patients with complex needs. ${ }^{3}$ However, it is less clear that deep sedation delivers comparable value to moderate sedation for routine colonoscopy when this strategy costs over $40 \%$ more than moderate sedation. ${ }^{7}$ Despite the added cost, deep sedation does not provide significantly improved safety, effectiveness or efficiency for routine colonoscopy. ${ }^{8}$ Addressing the appropriate use of deep sedation for routine colonoscopy will require additional research directed at addressing barriers to change.

Though both moderate and deep sedation provide an excellent patient experience, deep sedation provides a small increase in patient satisfaction, ${ }^{9}$ and patients and providers who highly value this may resist 
efforts to encourage judicious use of deep sedation. This may happen by two mechanisms: patients may themselves desire deep sedation, and clinicians may perceive patients to desire deep sedation. Strategies to reduce the use of deep sedation that fail to acknowledge both of these factors may be ineffective or, worse, harmful by deterring those who would benefit from colonoscopy from undergoing the procedure. ${ }^{10}$ For jurisdictions where deep sedation is uncommon, clinicians should nonetheless be striving to maximise the quality of the patient experience of colonoscopy, and they stand to benefit from evidence to support efforts to do so.

Fortunately, non-pharmacological interventions to improve the patient experience exist. Such interventions include preprocedural information, altering the patient's starting position, water exchange colonoscopy, audio and/or visual distraction and use of an ultrathin colonoscope. ${ }^{11-16}$ However, the breadth and effectiveness of these non-pharmacological interventions are not well described in the existing literature. The British Society of Gastroenterology's position statement on patient experience during gastrointestinal (GI) endoscopy affirms not only patient experience as a core dimension of healthcare quality but also enumerates few options available to endoscopists to alter the patient experience during GI endoscopy. ${ }^{17}$ To inform efforts to improve the patient experience of colonoscopy and potentially reduce the use of deep sedation for routine colonoscopy, additional research is needed.

To address this knowledge gap, we will conduct a systematic review to identify and synthesise the existing literature describing non-pharmacological interventions to improve the patient experience of colonoscopy.

\section{METHODS AND ANALYSIS}

This systematic review protocol was prepared in accordance with the Preferred Reporting Items for Systematic review and Meta-Analysis Protocols (PRISMA-P) statement. ${ }^{18}$ This review was prospectively registered in PROSPERO and will be reported in accordance with the PRISMA statement. ${ }^{19}$ Significant amendments to this protocol will be reported.

\section{Patient and public involvement}

Patients and the public were not involved in the design, conduct or reporting of this study. Results of the systematic review will be disseminated to patients and the public through relevant conferences, peer-reviewed publications and social media.

\section{Identifying the research question}

The research question was identified in consultation with clinicians and researchers with expertise in colonoscopy and a preliminary literature search. The research question is as follows: what (if any) non-pharmacological interventions are effective compared with placebo or usual care for improving the patient experience with routine colonoscopy under no or moderate sedation?

\section{Eligibility criteria}

Peer-reviewed publications in English of randomised controlled trials will be eligible for inclusion. Studies, including adults $>18$ years old evaluating the effectiveness of any non-pharmacological intervention (given within 1 week of the date of colonoscopy) compared with placebo or usual care for improving the patient experience of colonoscopy (measured within 24 hours of discharge from the endoscopy suite) under moderate or no sedation will be included.

\section{Exclusion criteria}

Due to resource constraints, we will exclude studies published in languages other than English. This practice is unlikely to introduce bias. ${ }^{20}$ We will exclude studies of non-elective colonoscopies and colonoscopies in patients admitted to hospital if these are the only patients who were randomised. Studies not reporting any outcomes of interest will also be excluded.

We anticipate that our broad search will identify a large number of publications and have planned a threestage screening process to address this. Two independent reviewers will conduct a title screen, followed by an abstract screen and finally a full-text screen using piloted forms on the DistillerSR platform (Evidence Partners, Ottawa, Canada). Disagreements will be resolved through discussion and consensus, involving a third reviewer if necessary.

\section{Information sources}

A search strategy will be designed with assistance from an expert information specialist. The search strategy will be reviewed using the Peer Review of Electronic Search Strategies checklist. ${ }^{21}$ The search terms include variations on terms for 'Colonoscopy' and 'Patient Comfort' (online supplemental file). MEDLINE, Embase, CINAHL, PSYCINFO, Scopus and the Cochrane Central Register of Controlled Trials will be searched for publications from inception until 2020, with an additional search prior to publication. In addition to these databases, reference lists of included articles, reference lists of relevant systematic reviews, clinicaltrials.gov, a PubMed Related Article search and OpenGrey will be searched.

\section{Data extraction}

We will use piloted forms developed in Microsoft Excel (Microsoft, Santa Rosa, California, USA) to extract study details (title, author, year of publication, location, setting (hospital/clinic) and source of funding), study design (duration, outcome measure(s), type of sedation used, inclusion and exclusion criteria, sample size and number of endoscopists participating in the trial), participant characteristics (patient demographics, including age, sex and indication for colonoscopy) and intervention characteristics (device or technique, intervention timing and duration). 
Intention-to-treat effect estimates and all measures of dispersion will be extracted. Where any study data are missing, authors will be contacted for clarification. All data will be extracted by two independent reviewers. Any disagreements will be resolved through discussion and consensus, involving a third reviewer if necessary.

\section{Outcomes and prioritisation}

A preliminary literature review has shown heterogeneity in the types of instruments used to assess the patient experience and there is no existing consensus on how to prioritise measures of patient experience. ${ }^{17}{ }^{22}$ Therefore, studies reporting any quantitative measure of patient experience, including satisfaction, anxiety, pain or discomfort as an outcome, will be included. Secondary outcomes will include willingness to repeat the procedure, adenoma detection rate, polyp detection rate, caecal intubation rate, caecal intubation time, total colonoscopy time, endoscopist satisfaction, cost in dollars and the occurrence of any adverse events (eg, bleeding and perforation). Studies reporting no outcomes of interest will be excluded.

Patient experience, the primary outcome, will be reported as a continuous outcome expressed as standardised mean difference between treatment and control arms given that we expect heterogeneity in the types of instruments used to assess the patient experience. ${ }^{23}$ Secondary outcomes will be expressed as follows: willingness to repeat the procedure will be a binary outcome expressed as relative risk; adenoma detection rate, polyp detection rate, caecal intubation rate and occurrence of adverse events (eg, bleeding and perforation) will be reported as relative risks and cecal intubation time, total colonoscopy time, endoscopist satisfaction and cost will be reported as mean differences.

\section{Quality assessment}

Risk of bias for each outcome reported by included studies will be assessed by two independent reviewers using the Cochrane Risk of Bias Tool V.2.0. ${ }^{24}$

\section{Data synthesis}

We will first present a descriptive summary of trial characteristics, including study design, intervention characteristics and participant characteristics. Where $\geq 3$ studies examine a single intervention, we will conduct quantitative synthesis by using pairwise inverse-variance randomeffects meta-analysis to estimate the pooled standardised mean difference between the intervention and control for all continuous primary and secondary outcomes and relative risk for binary outcomes. ${ }^{23}$ Binary outcomes will also be reported as number needed to treat/harm. If a study arm experiences no events, 0.5 will be added to each cell of the $2 \times 2$ table to facilitate synthesis. We will report 95\% CIs for all measures of average treatment effect, as well as prediction intervals where appropriate. ${ }^{25}$ Studies missing summary data (eg, variance and sample size) will be dealt with on a case-by-case basis to determine if the summary data can be approximated; otherwise, they will be excluded from quantitative synthesis and summarised narratively. In either case, the implications of the missing data will be discussed.

We will use $\mathrm{I}^{2}$ scores to quantify heterogeneity, with $0 \%-25 \%$ indicating low heterogeneity, $25 \%-50 \%$ indicating moderate heterogeneity and $>50 \%$ indicating high heterogeneity. ${ }^{26}$ If sufficient data are available, we will explore heterogeneity in pooled estimates of effect by conducting univariable random-effects meta-regression on critical trial characteristics defined a priori: risk of bias (low vs high/some concerns as classified by the Cochrane Risk of Bias Tool) and study size $>100$ patients. To identify publication bias resulting from missing studies, funnel plots will be constructed if more than 10 studies are identified for inclusion. Two-sided $\mathrm{p}<0.05$ will indicate statistical significance. Analyses will be performed using Review Manager V.5.0 (Cochrane Collaboration, Oxford, UK) and the meta and metafor packages in RStudio (RStudio, Boston, Massachusetts, USA). ${ }^{27}$ Where there are $<3$ studies or studies are too heterogeneous to be quantitatively synthesised, we will conduct a narrative synthesis. Studies incorporated in narrative synthesis will be grouped by the type of intervention that they investigate and qualitatively compared within those groups.

\section{CONFIDENCE IN CUMULATIVE EVIDENCE}

Two reviewers will independently assess confidence in the cumulative body of evidence using the Grading of Recommendations Assessment, Development and Evaluation (GRADE) framework for each identified nonpharmacological intervention. Discrepancies will be resolved through discussion and mediation by a third reviewer if necessary. The quality of evidence will be graded as high, medium, low or very low.

\section{ETHICS AND DISSEMINATION}

Ethics approval is not required for this study of preexisting literature.

\section{DISCUSSION}

The proposed systematic review will identify and assess non-pharmacological interventions to improve the patient experience of colonoscopy under moderate or no sedation.

This review builds on a previous review by Leung who attempted to describe the breadth of interventions available to endoscopists to improve the patient experience. ${ }^{28}$ Leung's review, while successful at identifying several methods to reduce discomfort during colonoscopy, is of limited applicability to the present context. The review included studies of patients undergoing colonoscopy with deep or moderate sedation, searched only a single database and is now over 10 years old. Other reviews have assessed the effectiveness of specific interventions 
with patient experience as an outcome. ${ }^{29} 30$ However, these have focused on single interventions, and evidence syntheses are absent for many interventions relevant to the study question. ${ }^{13-15} 3132$ In contrast, this contemporary review is designed to search multiple electronic databases, use standardised tools to assess study quality, quantitatively synthesise study results where possible, explores heterogeneity using a pre-specified approach and specifically addresses colonoscopy under moderate/ no sedation. ${ }^{28}$

By synthesising the evidence supporting the use of nonpharmacological interventions to improve the patient experience, clinicians may be empowered to look beyond the use of sedatives and analgesics and take a holistic approach to optimising the patient experience of colonoscopy. With patient experience being a key dimension of colonoscopy quality, improving the patient experience of colonoscopy is, in itself, a desirable outcome of synthesising evidence and increasing awareness about nonpharmacological interventions.

Additionally, the findings of our study will be of particular relevance to jurisdictions facing increasing costs of colonoscopy secondary to increased use of deep sedation. One potential driver of deep sedation use is the small patient satisfaction advantage it confers over moderate sedation ${ }^{8}$ - synthesising the evidence and raising awareness around non-pharmacological interventions to improve the patient experience may reduce dependence on deep sedation to optimise the patient experience for routine colonoscopy.

Lastly, as the world grapples with the COVID-19 pandemic and many jurisdictions are pausing elective endoscopy, there is the possibility that clinicians may face resource shortages and rationing when elective endoscopy resumes. By synthesising the evidence around options to improve the patient experience of colonoscopy, we may aid clinicians in adapting to these potential resource constraints.

This review has limitations. Some interventions may be evaluated by few studies, and others may be evaluated by studies that are heterogeneous in the outcome measures, patient populations and implementation of the intervention. These challenges may pose barriers to quantitative synthesis. Recognising this, we have pre-specified conditions for conducting quantitative synthesis and methods for exploring heterogeneity. Even if quantitative synthesis is not possible for many interventions, a narrative review will still provide a useful map of the current body of literature supporting non-pharmacological interventions to improve the patient experience of colonoscopy and future directions for research.

\footnotetext{
Author affiliations

${ }^{1}$ Department of Surgery, Unity Health Toronto, Toronto, Ontario, Canada

${ }^{2}$ Institute of Health Policy, Management and Evaluation, University of Toronto, Toronto, Ontario, Canada

${ }^{3}$ Sunnybrook Health Sciences Centre, Toronto, Ontario, Canada

${ }^{4}$ Cancer Care Ontario, Toronto, Ontario, Canada
}

${ }^{5}$ Scotiabank Health Sciences Library, Li Ka Shing Knowledge Institute, Toronto, Ontario, Canada

${ }^{6}$ Melbourne School of Population and Global Health, Melbourne, Victoria, Australia

Contributors All authors (CS-C-L, MC, JT, DL, TK and NNB) contributed substantially to the design and conception of the study, drafted the manuscript and revised it critically for important intellectual content, approved the final submission and agree to be held accountable for all aspects of the work. CS-C-L and NNB conceived the initial study and all authors (CS-C-L, MC, JT, DL, TK and NNB) contributed to the development of the study protocol and inclusion criteria. TK developed the search strategy with input from all other authors.

Funding This work was supported by the Canadian Institutes of Health Research (ClHR) grant number FDN - 148470.

Competing interests None declared.

Patient and public involvement Patients and/or the public were not involved in the design, or conduct, or reporting, or dissemination plans of this research.

Patient consent for publication Not required.

Provenance and peer review Not commissioned; externally peer reviewed.

Open access This is an open access article distributed in accordance with the Creative Commons Attribution Non Commercial (CC BY-NC 4.0) license, which permits others to distribute, remix, adapt, build upon this work non-commercially, and license their derivative works on different terms, provided the original work is properly cited, appropriate credit is given, any changes made indicated, and the use is non-commercial. See: http://creativecommons.org/licenses/by-nc/4.0/.

ORCID iD

Colin Sue-Chue-Lam http://orcid.org/0000-0003-2732-3929

\section{REFERENCES}

1 Baxter N, Rabeneck L, Tinmouth J. Report on the Development of Practice Recommendations for the Use of Sedation for HospitalBased Routine Colonoscopies. Toronto, 2018.

2 Krigel A, Chen L, Wright JD, et al. Substantial increase in anesthesia assistance for outpatient colonoscopy and associated cost nationwide. Clinical Gastroenterology and Hepatology 2019;17:2489-96.

3 Early DS, Lightdale JR, Vargo JJ, et al. Guidelines for sedation and anesthesia in Gi endoscopy. Gastrointest Endosc 2018;87:327-37.

4 Rex DK, Schoenfeld PS, Cohen J, et al. Quality indicators for colonoscopy. Gastrointest Endosc 2015;81:31-53.

5 Kaminski MF, Thomas-Gibson S, Bugajski M, et al. Performance measures for lower gastrointestinal endoscopy: a European Society of gastrointestinal endoscopy (ESGE) quality improvement initiative. Endoscopy 2017;49:378-97.

6 Armstrong D, Barkun A, Bridges R, et al. Canadian association of gastroenterology consensus guidelines on safety and quality indicators in endoscopy. Can J Gastroenterol 2012;26:17-31.

7 Pace D, Borgaonkar M. Deep sedation for colonoscopy is unnecessary and wasteful. Can Med Assoc J 2018;190:E153-4.

8 Dossa F, Dube C, Tinmouth J, et al. Practice recommendations for the use of sedation in routine hospital-based colonoscopy 2019.

9 Singh $\mathrm{H}$, Poluha W, Cheang M, et al. Propofol for sedation during colonoscopy. Cochrane Database Syst Rev 2008;50.

10 McLachlan S-A, Clements A, Austoker J. Patients' experiences and reported barriers to colonoscopy in the screening context-A systematic review of the literature. Patient Educ Couns 2012;86:137-46.

11 Cadoni S, Sanna S, Gallittu P, et al. A randomized, controlled trial comparing real-time insertion pain during colonoscopy confirmed water exchange to be superior to water immersion in enhancing patient comfort. Gastrointest Endosc 2015;81:557-66.

12 Bechtold ML, Perez RA, Puli SR. Effect of music on patients undergoing outpatient colonoscopy. WJG 2006;12:7309-12.

13 Vergis N, McGrath AK, Stoddart CH, et al. Right or left in colonoscopy (ROLCOL)? A randomized controlled trial of right- versus left-sided starting position in colonoscopy. $\mathrm{Am} \mathrm{J}$ Gastroenterol 2015;110:1576-81.

14 Sato K, Ito S, Kitagawa T, et al. A prospective randomized study of the use of an ultrathin colonoscope versus a pediatric colonoscope in sedation-optional colonoscopy. Surg Endosc 2017;31:5150-8.

15 Garborg K, Løberg M, Matre J, et al. Reduced pain during screening colonoscopy with an ultrathin colonoscope: a randomized controlled trial. Endoscopy 2012;44:740-6. 
16 Pearson S, Maddern GJ, Hewett P. Interacting effects of preoperative information and patient choice in adaptation to colonoscopy. Dis Colon Rectum 2005;48:2047-54.

17 Rees CJ, Trebble TM, Von Wagner C, et al. British Society of gastroenterology position statement on patient experience of $\mathrm{Gi}$ endoscopy. Gut 2020;69:1-2.

18 Moher D, Shamseer L, Clarke M, et al. Preferred reporting items for systematic review and meta-analysis protocols (PRISMA-P) 2015 statement. Syst Rev 2015;4:1-9.

19 Moher D, Liberati A, Tetzlaff J, et al. Preferred reporting items for systematic reviews and meta-analyses: the PRISMA statement. PLoS Med 2009;6:e1000097.

20 Morrison A, Polisena J, Husereau D, et al. The effect of Englishlanguage restriction on systematic review-based meta-analyses: a systematic review of empirical studies. Int J Technol Assess Health Care 2012;28:138-44.

21 McGowan J, Sampson M, Salzwedel DM, et al. PRESS Peer Review of Electronic Search Strategies: 2015 Guideline Statement. J Clin Epidemiol 2016;75:40-6.

22 Brown S, Bevan R, Rubin G, et al. Patient-Derived measures of Gi endoscopy: a meta-narrative review of the literature. Gastrointest Endosc 2015;81:1130-40.

23 Fu R, Vandermeer BW, Shamliyan T, et al. Methods Guide for Comparative Effectiveness Reviews Handling Continuous Outcomes in Quantitative Synthesis. Rockville, MD: AHRQ, 2013. www. effectivehealthcare.ahrq.gov/reports/final.cfm
24 Higgins J, Savović J, Page MJ, et al. Rob 2: a revised Cochrane risk-of-bias tool for randomized trials. Cochrane methods, 2019. Available: https://methods.cochrane.org/

25 Riley RD, Higgins JPT, Deeks JJ. Interpretation of random effects meta-analyses. BMJ 2011;342:d549-967.

26 Higgins J, Green S. Cochrane Handbook for systematic reviews of interventions version 5.1.0. The Cochrane Collaboration, 2011. www. handbook.cochrane.org

27 Viechtbauer W. Conducting meta-analyses in R with the metafor package 2010.

28 Leung FW. Methods of reducing discomfort during colonoscopy. Dig Dis Sci 2008;53:1462-7.

29 Fuccio L, Frazzoni L, Hassan C, et al. Water exchange colonoscopy increases adenoma detection rate: a systematic review with network meta-analysis of randomized controlled studies. Gastrointest Endosc 2018;88:589-97.

30 Bechtold ML, Puli SR, Othman MO, et al. Effect of music on patients undergoing colonoscopy: a meta-analysis of randomized controlled trials. Dig Dis Sci 2009;54:19-24.

31 Hu P-H, Peng Y-C, Lin Y-T, et al. Aromatherapy for reducing colonoscopy related procedural anxiety and physiological parameters: a randomized controlled study. Hepatogastroenterology 2010;57:1082-6.

32 Amer-Cuenca JJ, Goicoechea C, Girona-López A, et al. Pain relief by applying transcutaneous electrical nerve stimulation (TENS) during unsedated colonoscopy: a randomized double-blind placebocontrolled trial. Eur J Pain 2011;15:29-35. 